\section{Peter Minder}

Am 31. Juli 1986 wurde, im Anschluss an eine davor im Juni mit grossem Erfolg durchgeführte erste Silserwoche, in Wohlen/AG die "Schweizerische Aerztegesellschaft für Homöopathie und Phytotherapie», wie die SAHP damals geheissen hat, gegründet.

Teilgenommen an der Gründungsversammlung haben der Präsident Dr. med. Martin Furlenmeier, der Vizepräsident Dr. med. Clemens Dietrich und der Aktuar und Kassier Dr. med. Urs Steiner, der nach einem Schreiben Martin Furlenmeiers «ihm in allen bisherigen Kursen angenehm aufgefallen war». Diese drei Vorstandsmitglieder waren zugleich die ersten drei ordentlichen Mitglieder der Gesellschaft; als Revisor war an der Gründungsversammlung der Chemiker Konrad Imhof anwesend, der mit dem Institut für medizinische Fortbildung (IMF) die Kursorganisation übernahm.

\title{
Die SAHP: 25 Jahre Vereinsgeschichte
}

Eine erste Generalversammlung fand am 9. April 1987 in Luzern statt; anwesend waren 29 von insgesamt 36 Mitgliedern, die laut Statuten allesamt ausserordentliche Mitglieder waren, da sie den 2-Jahres-Kurs noch nicht abgeschlossen hatten.

Am 23. April 1987 wurden dann auf Rigi-Kaltbad nach Absolvierung der 22 Ganztageskurse 16 Ärzte in die ordentliche Mitgliedschaft aufgenommen.

Die Ausbildung unter Dr. med. Martin Furlenmeier war geprägt von seinem eindrücklich breiten Wissen in Gebieten der Botanik, der Phytotherapie, der Physik und der Chemie, kombiniert mit einem charismatischen Feuer für die Homöopathie, mit dem er die Kursteilnehmer zu begeistern vermochte. Dem Zeitgeist entsprechend war die Ausbildung ganz auf den kentianischen Prinzipien basiert. Unterstützt wurde sie durch die mit philosophischen und weltan- schaulichen Aspekten gespickten Referate des Vizepräsidenten Clemens Dietrich.

1990 wurde der Vertrag mit Herrn Imhof gekündigt; seit diesem Zeitpunkt wird das Sekretariat und Kurswesen bravourös von Frau Vreni Greising organisiert.

1992 kam es anlässlich der Generalversammlung in Giessbach zu einem Eklat - aufgrund der Unzufriedenheit des Präsidenten mit einer erarbeiteten Studienordnung trennte man sich von Dr. med. Clemens Dietrich; als neuer Vizepräsident brachte nun Dr. med. Klaus Henning Gypser die für die SAHP wohl entscheidende Wende mit der Fokussierung der Ausbildung auf die Methode nach Bönninghausen.

1994 veranlasste eine schwere Erkrankung Dr. med. Martin Furlenmeier, sein Amt als Präsident der SAHP niederzulegen. Sein Lebenswerk sowie sein Engagement für die

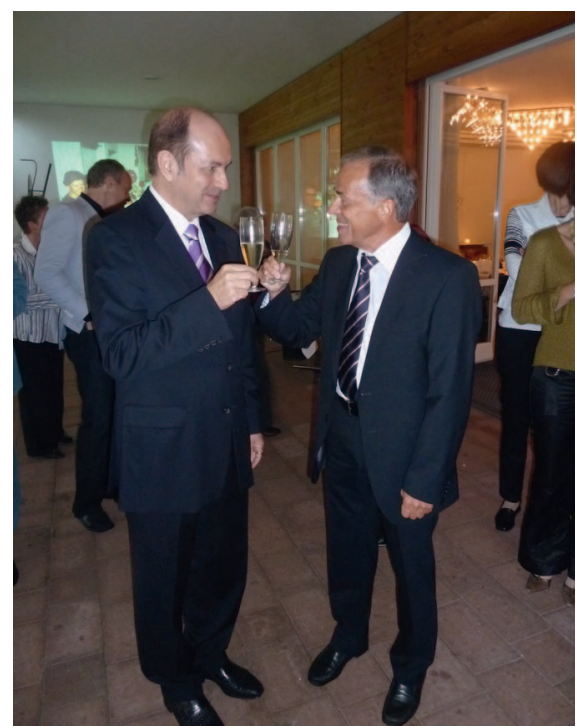

Abb. 2. Die beiden Gründungsmitglieder Dr. med. Urs Steiner und Dr. med. Clemens Dietrich stossen auf 25 Jahre SAHP an.

Abb. 1. Die aufmerksame Zuhörerschaft während des Vortrags «Materia Medica Rhododendron». 
Homöopathie und insbesondere für die Herstellung von einwandfreien homöopathischen Arzneien wurde anlässlich der Generalversammlung im Hotel Montana in Luzern von Dr. med. Urs Steiner gewürdigt.

Dr. med. Urs Steiner übernahm das Präsidium der SAHP - die sich mangels des grossen Phytotherapiewissens von Martin Furlenmeier von nun an «Aerztegesellschaft für HomöoPathie» nannte. Er trat kein leichtes Erbe an. In seiner Amtszeit gelang es ihm, die monarchisch geführte Gesellschaft, die von einem Mann und seiner Gattin geprägt war, zu demokratisieren. Der Vorstand wurde erweitert und mehrere Referenten teilten sich die Aufgabe der Aus- und Fortbildung und ergänzten sich gegenseitig in ihren persönlichen Vorlieben und Schwerpunkten. Die relativ starren Gesellschaftsstrukturen wurden weicher; es war beispielsweise nicht selbstverständlich, mit den anderen Kursteilnehmern und besonders mit dem Kursleiter per Du zu sein. Die Apotheker, unter Dr. med. Martin Furlenmeier eher nur geduldet, wurden zu einem zunehmend wichtigen Standbein der SAHP. Trotzdem wur-

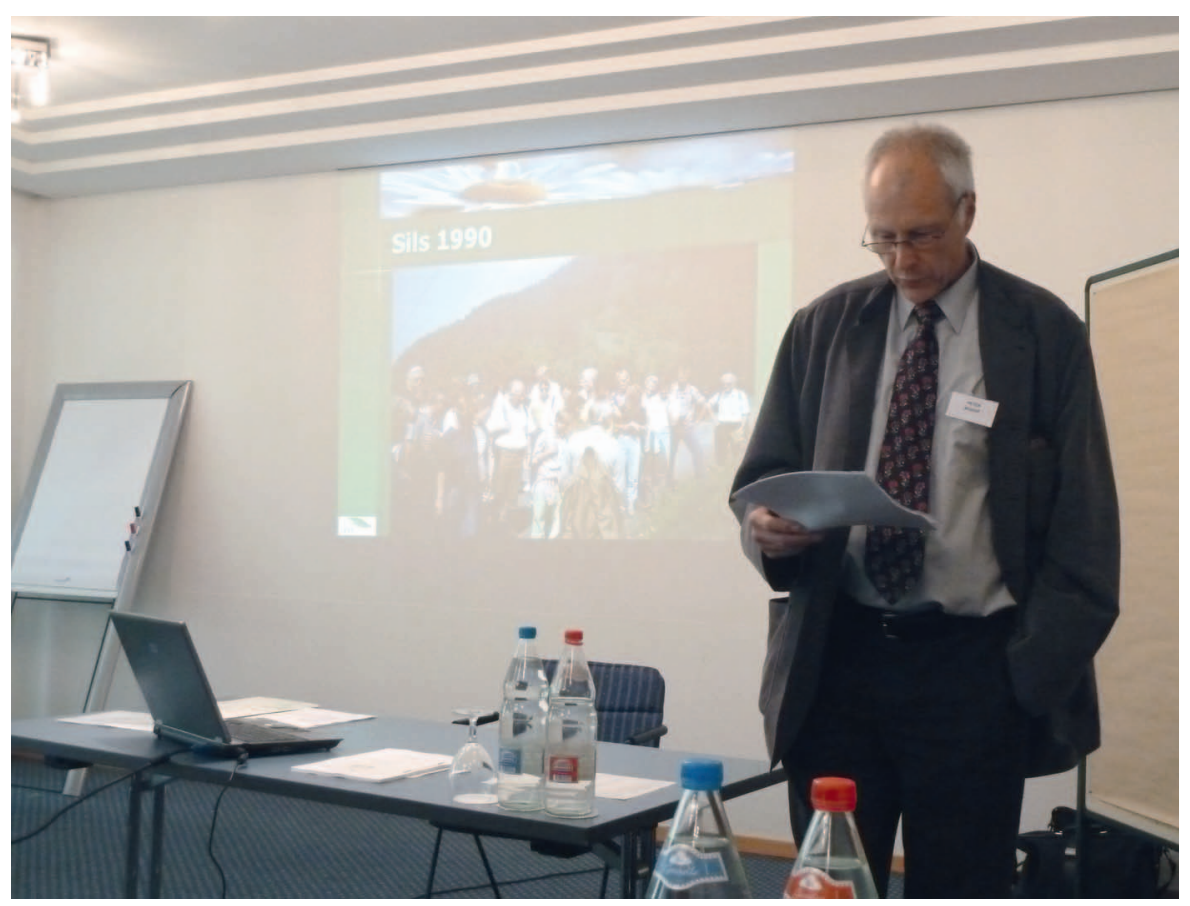

Abb. 3. Dr. med. Peter Minder beim geschichtlichen Rückblick. den die guten Traditionen weitergeführt; die Silserwoche, die bis anhin ein Bestandteil des Grundlehrgangs gewesen war und demzufolge nur zweimal besucht werden konnte, wurde ab 1994 in einem neuen Modus nun jährlich für alle Mitglieder angeboten.

$\mathrm{Zu}$ diesem Zeitpunkt übernahm Dr. pharm. Beat Wittwer erstmals die Verantwortung für die botanische Exkursion. Dr. med. Martin Furlenmeier durfte endlich loslassen und verabschiedete sich für immer von uns. Die neu konzipierte Silserwoche bildete, nebst qualitativ wertvoller Fortbildung, fortan ein wichtiges Element zur Förderung von Freundschaft und «Kittung» der Gesellschaft.

Konsequent weitergeführt wurde die Ausbildung auf der Grundlage der Bönninghausen-Methode, unterstützt durch das von Urs Steiner geschaffene Softwareprogramm "Amokoor» und ergänzt durch seine Ausführungen über Elemente der Chaostheorie aus den Erkenntnissen der neuesten Teilchenphysik.

Im Jahre 2001, nach 15-jähriger Vorstandstätigkeit und 7-jährigem Präsidium, konnte er dann eine gefestigte, in sich stimmige Gesellschaft

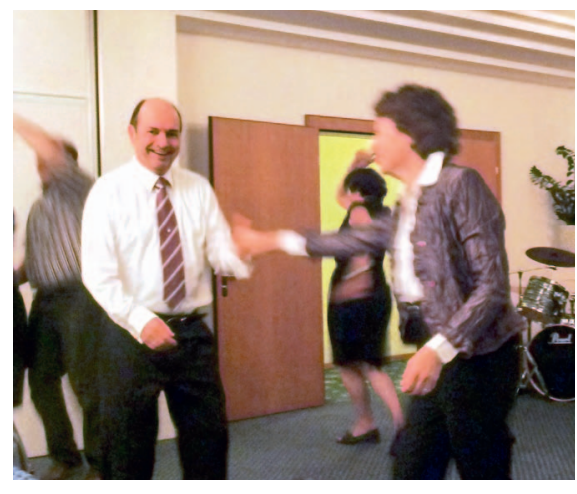

Abb. 4. Fröhlicher Ausklang bei guter Stimmung und Tanz.

in die Hände von Dr. med. Heiner Frei als neuen Präsidenten geben.

Mit ihm übernahm ein herausragender homöopathischer Praktiker die SAHP. Er strukturierte den 3-jährigen Grundausbildungsgang neu, widmete sich intensiv den Möglichkeiten der Effizienzsteigerung in der täglichen Praxis und vermittelte seine Ergebnisse in fesselnden Kursen. Er lernte bei Dr. med. Klaus Henning Gypser die Möglichkeit der Polaritätsanalyse, die auf Bönninghausen zurückgeht, kennen und entwickelte diese zusammen mit anderen Vorstandsmitgliedern der SAHP zu einem präzisen Arbeitsinstrument, das inzwischen unter dem Namen «FreiMethode» Bekanntheit erlangt hat. In seine Amtszeit fällt mit 171 auch der höchste Mitgliederstand im Jahre 2003.

Den Höhepunkt seiner Präsidialkarriere bildete aber, nebst der von Dr. med. Urs Steiner geleiteten Kilimandscharo-Besteigung, der erfolgreiche Abschluss der ADHS-Studie in Zusammenarbeit mit der Universität Bern, mit der er die Wirksamkeit der Homöopathie bei diesem Krankheitsbild belegen konnte. Leider viel $\mathrm{zu}$ früh musste er dann sein Amt aus persönlichen Gründen im Jahre 2005 bereits wieder niederlegen.

Seit 2005 steuert Dr. med. Peter Minder das SAHP-Boot. Nur 2 Monate nach seiner Wahl bläst der Gesellschaft mit dem Entscheid von Bun- 
desrat Couchepin, der Streichung der homöopathischen Leistungen aus der Grundversicherung, ein kalter Wind entgegen. Dr. med. Peter Minder versucht seither, die SAHP auf rauer See auf Kurs zu halten, unterstützt von den Vizepräsidenten - zuerst Dr. med. Julia Brönnimann und Beat Wittwer, seit 2008 Jacqueline Ryffel - und dem übrigen Vorstand. Rau sind auch die stetig gezielten Angriffe in den Medien sowie der Nachlass des Publikumsinteresses im Rahmen zyklischer Abläufe. Dies alles hat logischerweise auch $\mathrm{zu}$ einem zeitweiligen Rückgang der Kursbesuche, insbesondere der Grundausbildung, geführt.

Dr. med. Peter Minder hat in seiner Amtszeit bis jetzt den Schwerpunkt aufdie Vermittlung von theoretischen
Grundlagen der Homöopathie gelegt: die Arbeitsweise von C.M. Boger als Erweiterung und nahtlosen Anschluss an die Bönninghausen-Methode und diefundierte Bearbeitung der Materia Medica.

Was die erneute Aufnahme der homöopathischen Leistungen in die Grundversicherung bringen wird, bleibt abzuwarten; erste positive Tendenzen sind sichtbar. Am letzten 5-tägigen Einführungskurs waren wieder über 20 Teilnehmer anwesend. Das Interesse ist wieder wesentlich grösser. Die SAHP wird weitermachen im steten Bestreben, die Homöopathie auf qualitativ hohem Niveau zu fördern und weiterzugeben, die Freundschaften zu pflegen und die Gesellschaft als Untergruppe ihres Dachverbands, des Schweizerischen

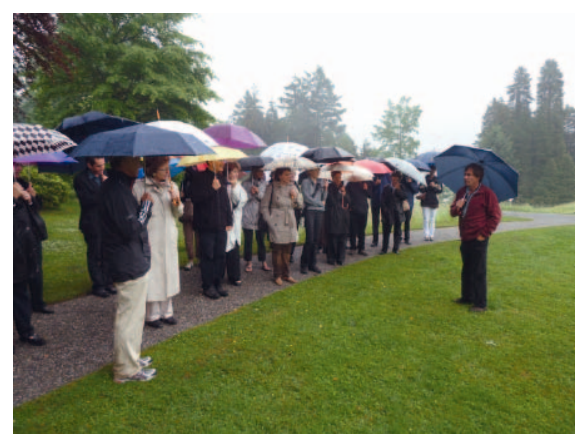

Abb. 5. Führung im Schlossgarten Buonas.

Vereins Homöopathischer Aerztinnen und Aerzte (SVHA), weiterzuführen.

Und last but not least: Der heutige Präsident des SVHA, Dr. med. Clemens Dietrich, ist seit längerem auch wieder Mitglied und Referent der SAHP. 